Supporting Information for

\title{
Seismic gaps and intraplate seismicity around Rodrigues Ridge (Indian Ocean) from time-domain array analysis
}

Manvendra Singh ${ }^{1,2}$ and Georg Rümpker ${ }^{2}$

$5{ }^{1}$ Mauritius Oceanography Institute, Avenue des Anchois, Morcellement de Chazal, Albion, Mauritius

${ }^{2}$ Institute of Geosciences, Goethe-University Frankfurt, Frankfurt am Main, Germany

Correspondence to: Manvendra Singh (msingh@moi.intnet.mu)

10

\section{Contents of this file}

Figures $\mathrm{S} 1$ and $\mathrm{S} 2$

Tables S1 and S2

15

\section{Introduction}

Figure S1 shows the influence of the model parameters on the determination of the epicentral distance for an event that occurs at a distance of $\sim 120 \mathrm{~km}$ from the array.

20 Figure S2 shows the influence of the model parameters on the determination of the epicentral distance for an event that occurs at a distance of $\sim 265 \mathrm{~km}$ from the array.

Table S1 provides detailed information of the events located using the array deployed on the island of Rodrigues.

25

Table S2 gives details of the events in each of the seismic clusters observed. 


\section{Cluster 1}
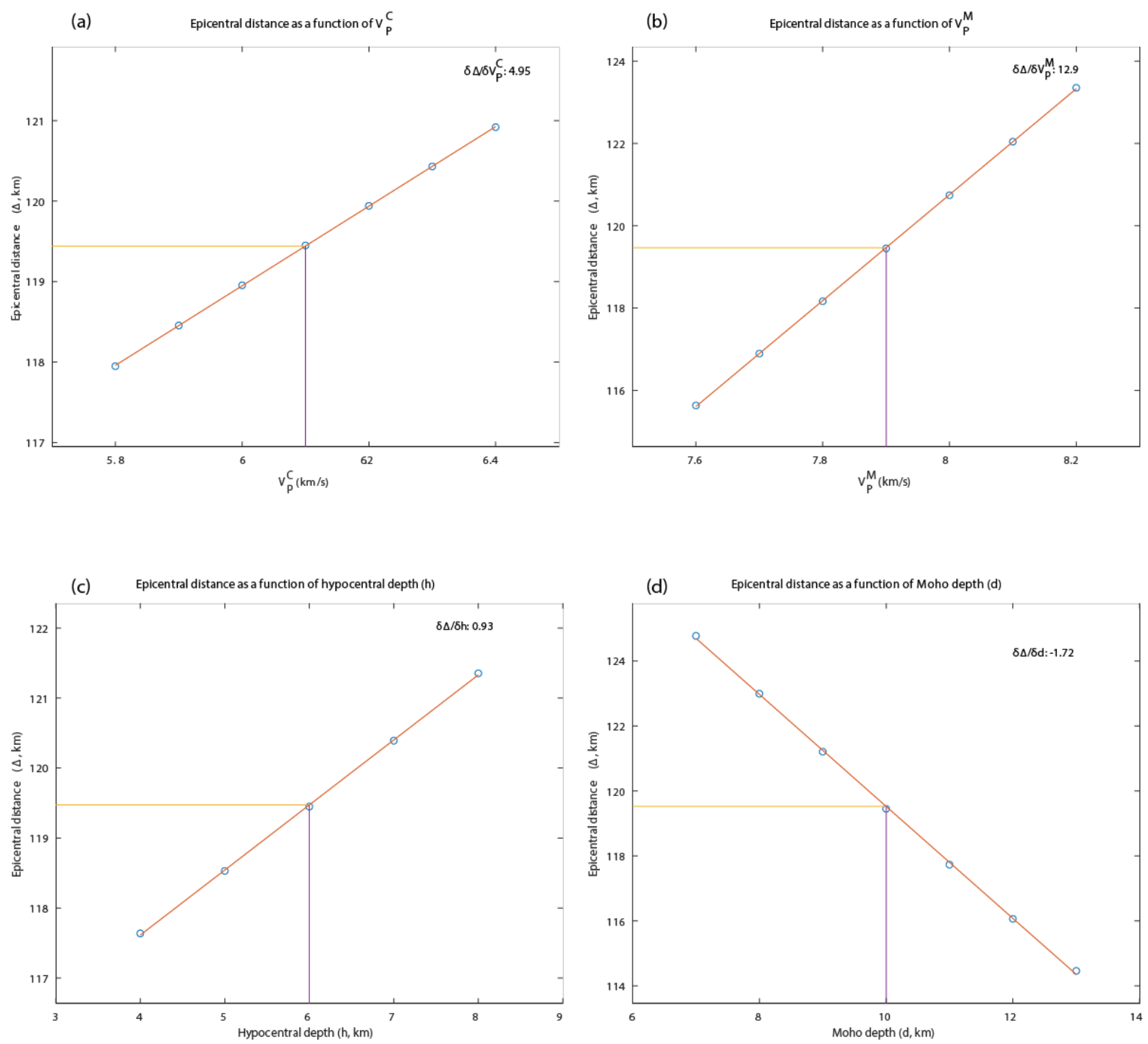

Figure S1. Influence of model parameters on the epicentral distance for events at a distance of $\sim 140 \mathrm{~km}$. Changes are shown for (a) the crustal P-wave velocity $\left(V_{\mathrm{P}}^{\mathrm{C}}\right)$, (b) the P-wave velocity in the mantle $V_{\mathrm{P}}^{\mathrm{M}}$, (c) the hypocentral depth (h) and (d) the Moho depth (d). 


\section{Cluster 3}
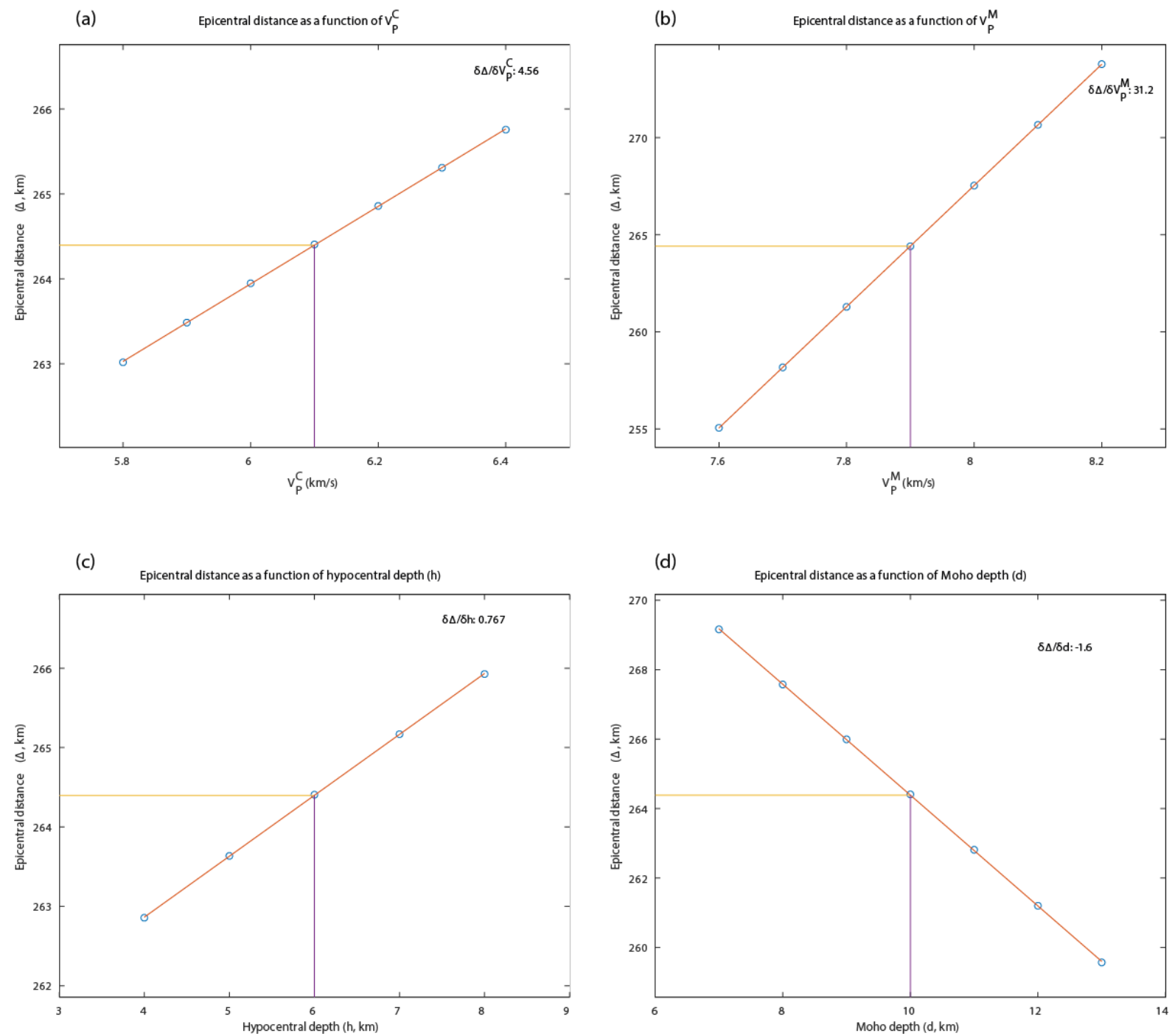

Figure S2. Influence of model parameters on the epicentral distance for events at a distance of 40 $\sim 265 \mathrm{~km}$. Changes are shown for (a) the crustal P-wave velocity $\left(V_{\mathrm{P}}^{\mathrm{C}}\right)$, (b) the P-wave velocity in the mantle $V_{\mathrm{P}}^{\mathrm{M}}$, (c) the hypocentral depth (h) and (d) the Moho depth (d). 


\begin{tabular}{|c|c|c|c|c|c|c|c|c|}
\hline \multirow{2}{*}{$\begin{array}{l}\text { Date,time } \\
\text { (yymmdd,hh:mm:ss) }\end{array}$} & \multirow[t]{2}{*}{ Lat. ( $\left.{ }^{\circ}\right)$} & \multirow{2}{*}{$\begin{array}{l}\text { Lon. } \\
\left({ }^{\circ}\right)\end{array}$} & \multirow[t]{2}{*}{$M_{\mathrm{L}}$} & \multirow[t]{2}{*}{ Dist. \pm SD (km) } & \multirow{2}{*}{$\begin{array}{l}\text { BAZ } \\
\left({ }^{\circ}\right)\end{array}$} & \multicolumn{2}{|c|}{ BAZ error } & \multirow{2}{*}{$\begin{array}{l}v_{\mathrm{a}} \\
(\mathrm{km} / \mathrm{s})\end{array}$} \\
\hline & & & & & & $\begin{array}{l}\text { Min. } \\
\left({ }^{\circ}\right)\end{array}$ & $\begin{array}{l}\text { Max. } \\
(\circ)\end{array}$ & \\
\hline 141104,12:18:31 & -20.512 & 65.101 & $3.5 \pm 0.3$ & $194.46 \pm 3.14$ & 117.55 & 113.45 & 122.68 & 8.186 \\
\hline $141125,00: 53: 54$ & -22.083 & 64.061 & $3.3 \pm 0.2$ & $271.46 \pm 2.61$ & 166.50 & 164.17 & 167.94 & 8.600 \\
\hline $141127,01: 57: 58$ & -22.093 & 64.063 & $3.5 \pm 0.2$ & $272.58 \pm 2.88$ & 166.50 & 164.69 & 167.69 & 8.600 \\
\hline 150107,21:03:19 & -21.946 & 63.448 & $2.9 \pm 0.2$ & $248.59 \pm 1.35$ & 179.96 & 178.71 & 181.21 & 8.656 \\
\hline $150117,19: 24: 18$ & -20.217 & 65.022 & $2.7 \pm 0.0$ & $174.05 \pm 2.77$ & 109.13 & 108.38 & 111.75 & 8.344 \\
\hline 150202,21:07:05 & -20.791 & 65.060 & $2.3 \pm 0.1$ & $206.88 \pm 12.20$ & 125.78 & 123.28 & 129.23 & 9.154 \\
\hline 150212,22:08:04 & -19.076 & 62.273 & $2.2 \pm 0.1$ & $141.80 \pm 2.99$ & 299.66 & 297.65 & 301.21 & 8.036 \\
\hline $150214,08: 46: 45$ & -18.078 & 64.898 & $2.6 \pm 0.1$ & $237.25 \pm 4.41$ & 40.32 & 35.85 & 44.06 & 9.384 \\
\hline $150217,21: 43: 41$ & -19.117 & 62.277 & $2.4 \pm 0.3$ & $139.22 \pm 1.86$ & 298.12 & 296.10 & 299.66 & 7.974 \\
\hline $150217,22: 36: 07$ & -19.112 & 62.290 & $1.8 \pm 0.1$ & $138.24 \pm 2.54$ & 298.57 & 297.10 & 300.07 & 7.767 \\
\hline $150308,19: 02: 23$ & -20.579 & 64.350 & $1.8 \pm 0.0$ & $135.01 \pm 3.65$ & 135.82 & 133.21 & 139.34 & 8.845 \\
\hline $150310,20: 57: 35$ & -18.896 & 64.157 & $2.3 \pm 0.3$ & $117.39 \pm 2.08$ & 39.62 & 38.43 & 41.76 & 10.787 \\
\hline $150311,14: 46: 41$ & -18.924 & 64.187 & $2.0 \pm 0.4$ & $117.00 \pm 2.06$ & 41.76 & 38.43 & 42.88 & 10.818 \\
\hline $150321,10: 30: 34$ & -18.913 & 64.194 & $2.2 \pm 0.1$ & $118.49 \pm 2.55$ & 41.64 & 39.41 & 42.80 & 11.240 \\
\hline $150321,11: 41: 39$ & -18.918 & 64.193 & $3.0 \pm 0.3$ & $117.98 \pm 1.66$ & 41.76 & 39.62 & 42.88 & 10.818 \\
\hline $150322,19: 13: 48$ & -18.844 & 64.084 & $1.6 \pm 0.0$ & $117.38 \pm 1.60$ & 34.89 & 30.27 & 36.04 & 10.096 \\
\hline $150323,02: 08: 22$ & -18.918 & 64.159 & $2.1 \pm 0.4$ & $115.59 \pm 3.47$ & 40.43 & 39.18 & 42.71 & 11.448 \\
\hline $150324,23: 49: 14$ & -18.917 & 64.193 & $2.7 \pm 0.4$ & $118.05 \pm 2.40$ & 41.76 & 38.43 & 42.88 & 10.818 \\
\hline $150325,04: 36: 39$ & -18.913 & 64.194 & $2.1 \pm 0.1$ & $118.36 \pm 1.65$ & 41.64 & 39.41 & 42.71 & 11.24 \\
\hline $150325,09: 52: 03$ & -18.909 & 64.201 & $2.3 \pm 0.3$ & $119.20 \pm 1.59$ & 41.76 & 39.62 & 43.92 & 10.818 \\
\hline $150325,12: 53: 06$ & -18.768 & 63.971 & $2.2 \pm 0.3$ & $118.42 \pm 3.02$ & 27.84 & 25.96 & 43.88 & 9.977 \\
\hline $150327,17: 32: 44$ & -18.903 & 64.203 & $1.8 \pm 0.0$ & $119.91 \pm 1.66$ & 41.64 & 39.41 & 42.71 & 11.24 \\
\hline $150327,18: 28: 19$ & -18.913 & 64.194 & $1.6 \pm 0.0$ & $118.37 \pm 1.32$ & 41.64 & 38.43 & 42.80 & 11.24 \\
\hline $150328,18: 36: 13$ & -18.900 & 64.179 & $2.3 \pm 0.2$ & $118.54 \pm 1.42$ & 40.61 & 38.43 & 57.08 & 11.011 \\
\hline $150330,02: 12: 43$ & -18.923 & 64.218 & $2.8 \pm 0.2$ & $119.31 \pm 2.31$ & 42.88 & 39.62 & 45.00 & 10.627 \\
\hline $150331,01: 04: 36$ & -18.929 & 64.211 & $2.3 \pm 0.3$ & $118.32 \pm 2.27$ & 42.88 & 38.67 & 46.08 & 10.627 \\
\hline 150331,16:30:29 & -18.903 & 64.181 & $2.3 \pm 0.1$ & $118.37 \pm 2.52$ & 40.77 & 38.67 & 42.88 & 10.605 \\
\hline $150406,20: 25: 42$ & -18.870 & 64.156 & $3.0 \pm 0.3$ & $119.55 \pm 2.11$ & 38.67 & 36.88 & 41.88 & 10.57 \\
\hline $150407,13: 28: 36$ & -17.740 & 64.661 & $2.8 \pm 0.1$ & $253.75 \pm 5.94$ & 30.48 & 29.07 & 32.49 & 10.295 \\
\hline $150408,14: 54: 35$ & -18.548 & 65.115 & $2.2 \pm 0.0$ & $217.82 \pm 4.25$ & 53.87 & 50.19 & 57.33 & 8.867 \\
\hline $150408,15: 50: 50$ & -19.099 & 64.371 & $2.0 \pm 0.1$ & $118.49 \pm 1.54$ & 55.11 & 52.24 & 55.11 & 10.096 \\
\hline $150412,16: 15: 36$ & -18.917 & 64.190 & $2.4 \pm 0.2$ & $117.84 \pm 1.78$ & 41.64 & 40.61 & 42.80 & 11.240 \\
\hline $150413,23: 40: 29$ & -18.664 & 63.648 & $1.6 \pm 0.0$ & $118.28 \pm 1.91$ & 10.35 & 10.35 & 10.67 & 12.107 \\
\hline 150416,00:51:55 & -18.917 & 64.190 & $2.2 \pm 0.2$ & $117.83 \pm 1.67$ & 41.64 & 40.61 & 43.88 & 11.24 \\
\hline $150419,17: 46: 08$ & -22.086 & 64.006 & $3.0 \pm 0.2$ & $270.49 \pm 2.78$ & 167.69 & 165.33 & 169.11 & 8.641 \\
\hline $150422,02: 36: 21$ & -18.891 & 64.156 & $2.1 \pm 0.3$ & $117.73 \pm 2.73$ & 39.41 & 38.43 & 41.64 & 11.206 \\
\hline $150425,22: 12: 51$ & -18.886 & 64.161 & $1.6 \pm 0.1$ & $118.47 \pm 2.18$ & 39.41 & 38.43 & 40.61 & 11.206 \\
\hline $150506,17: 19: 15$ & -20.288 & 64.410 & $2.1 \pm 0.3$ & $120.42 \pm 1.51$ & 122.37 & 80.85 & 176.67 & 15.585 \\
\hline $150508,07: 19: 32$ & -22.006 & 64.042 & $3.3 \pm 0.3$ & $262.87 \pm 3.42$ & 166.21 & 164.69 & 167.69 & 8.781 \\
\hline $150511,21: 01: 30$ & -18.541 & 65.125 & $2.3 \pm 0.0$ & $220.12 \pm 2.09$ & 54.05 & 53.12 & 56.62 & 8.221 \\
\hline $150518,14: 40: 30$ & -18.364 & 64.764 & $3.0 \pm 0.2$ & $204.80 \pm 2.75$ & 43.21 & 38.10 & 46.85 & 8.970 \\
\hline $150518,17: 50: 30$ & -21.873 & 65.970 & $2.9 \pm 0.0$ & $356.70 \pm 2.02$ & 132.81 & 129.54 & 136.01 & 10.640 \\
\hline 150521,07:09:33 & -18.568 & 65.205 & $2.7 \pm 0.0$ & $225.23 \pm 2.91$ & 55.94 & 52.12 & 60.56 & 9.095 \\
\hline $150526,00: 24: 46$ & -23.453 & 64.345 & $3.0 \pm 0.1$ & $426.62 \pm 4.98$ & 167.42 & 165.02 & 169.87 & 8.824 \\
\hline $150604,12: 38: 18$ & -18.874 & 64.166 & $2.3 \pm 0.2$ & $120.64 \pm 1.73$ & 39.62 & 38.67 & 42.80 & 10.787 \\
\hline
\end{tabular}




\begin{tabular}{|l|l|l|l|l|l|l|l|l|}
\hline $150707,21: 57: 28$ & -18.851 & 65.329 & $2.5 \pm 0.2$ & $219.57 \pm 3.83$ & 64.52 & 59.46 & 67.26 & 8.731 \\
\hline $150730,02: 43: 14$ & -18.925 & 64.211 & $2.2 \pm 0.3$ & $118.58 \pm 2.04$ & 42.71 & 40.43 & 43.83 & 11.475 \\
\hline $151001,19: 22: 45$ & -18.882 & 64.164 & $2.2 \pm 0.0$ & $119.09 \pm 1.46$ & 39.41 & 38.43 & 41.64 & 11.206 \\
\hline $151012,20: 47: 05$ & -21.948 & 64.092 & $2.8 \pm 0.2$ & $257.66 \pm 2.97$ & 165.02 & 163.49 & 167.42 & 8.734 \\
\hline $151020,16: 51: 28$ & -19.179 & 65.825 & $2.6 \pm 0.0$ & $256.32 \pm 3.34$ & 77.06 & 74.19 & 80.51 & 8.249 \\
\hline $151114,20: 36: 38$ & -18.627 & 64.081 & $1.7 \pm 0.3$ & $137.74 \pm 0.87$ & 29.07 & 25.96 & 30.27 & 9.861 \\
\hline $151115,18: 42: 15$ & -18.491 & 65.077 & $2.6 \pm 0.1$ & $218.54 \pm 1.61$ & 51.90 & 48.46 & 54.60 & 8.640 \\
\hline $151130,16: 19: 41$ & -18.221 & 58.380 & $3.4 \pm 0.2$ & $557.87 \pm 5.53$ & 286.43 & 284.08 & 288.48 & 7.651 \\
\hline $151130,22: 27: 54$ & -21.952 & 64.108 & $2.9 \pm 0.2$ & $258.56 \pm 4.60$ & 164.69 & 163.13 & 167.42 & 8.919 \\
\hline $151211,17: 20: 39$ & -18.756 & 65.642 & $2.8 \pm 0.0$ & $253.78 \pm 13.46$ & 65.54 & 62.28 & 67.26 & 8.811 \\
\hline $151217,23: 27: 55$ & -18.403 & 65.045 & $2.8 \pm 0.2$ & $222.24 \pm 2.09$ & 49.39 & 46.73 & 51.90 & 8.811 \\
\hline $160212,19: 21: 45$ & -19.390 & 63.382 & $1.5 \pm 0.1$ & $36.27 \pm 1.09$ & 349.27 & 346.66 & 352.28 & 9.503 \\
\hline $160215,17: 29: 25$ & -18.778 & 63.993 & $1.3 \pm 0.0$ & $118.52 \pm 1.81$ & 29.07 & 25.96 & 39.62 & 9.861 \\
\hline $160330,21: 54: 15$ & -18.408 & 65.093 & $2.2 \pm 0.1$ & $225.67 \pm 4.41$ & 50.35 & 46.79 & 53.87 & 8.935 \\
\hline $160402,18: 13: 37$ & -18.449 & 65.041 & $2.8 \pm 0.1$ & $218.59 \pm 2.11$ & 50.35 & 47.64 & 53.87 & 8.935 \\
\hline $160412,19: 28: 01$ & -19.247 & 65.814 & $2.8 \pm 0.2$ & $253.54 \pm 5.78$ & 78.66 & 76.23 & 80.93 & 8.852 \\
\hline $160421,18: 15: 49$ & -17.650 & 62.573 & $2.0 \pm 0.0$ & $246.86 \pm 5.48$ & 337.99 & 336.68 & 339.34 & 10.178 \\
\hline $160503,21: 31: 33$ & -17.751 & 64.701 & $2.8 \pm 0.2$ & $254.84 \pm 4.73$ & 31.44 & 29.59 & 34.09 & 9.626 \\
\hline
\end{tabular}

Table S1. Details of the events detected and located using Rodrigues array. 


\begin{tabular}{|c|c|c|c|c|c|}
\hline $\begin{array}{l}\text { Date,time } \\
\text { (yymmdd,hh:mm:ss) }\end{array}$ & Lat. ( $\left.{ }^{\circ}\right)$ & Lon. ( $\left.{ }^{\circ}\right)$ & $M_{\mathrm{L}}$ & $\begin{array}{l}\text { Dist. } \pm \text { SD } \\
(\mathrm{km})\end{array}$ & $\mathrm{BAZ}\left({ }^{\circ}\right)$ \\
\hline \multicolumn{6}{|l|}{ Cluster 1} \\
\hline $150310,20: 57: 35$ & -18.896 & 64.157 & $2.3 \pm 0.3$ & $117.39 \pm 2.08$ & 39.62 \\
\hline $150311,14: 46: 41$ & -18.924 & 64.187 & $2.0 \pm 0.4$ & $117.00 \pm 2.06$ & 41.76 \\
\hline $150321,10: 30: 34$ & -18.913 & 64.194 & $2.2 \pm 0.1$ & $118.49 \pm 2.55$ & 41.64 \\
\hline $150321,11: 41: 39$ & -18.918 & 64.193 & $3.0 \pm 0.3$ & $117.98 \pm 1.66$ & 41.76 \\
\hline $150322,19: 13: 48$ & -18.844 & 64.084 & $1.6 \pm 0.0$ & $117.38 \pm 1.60$ & 34.89 \\
\hline 150323,02:08:22 & -18.918 & 64.159 & $2.1 \pm 0.4$ & $115.59 \pm 3.47$ & 40.43 \\
\hline $150324,23: 49: 14$ & -18.917 & 64.193 & $2.7 \pm 0.4$ & $118.05 \pm 2.40$ & 41.76 \\
\hline $150325,04: 36: 39$ & -18.913 & 64.194 & $2.1 \pm 0.1$ & $118.36 \pm 1.65$ & 41.64 \\
\hline $150325,09: 52: 03$ & -18.909 & 64.201 & $2.3 \pm 0.3$ & $119.20 \pm 1.59$ & 41.76 \\
\hline $150327,17: 32: 44$ & -18.903 & 64.203 & $1.8 \pm 0.0$ & $119.91 \pm 1.66$ & 41.64 \\
\hline $150327,18: 28: 19$ & -18.913 & 64.194 & $1.6 \pm 0.0$ & $118.37 \pm 1.32$ & 41.64 \\
\hline $150328,18: 36: 13$ & -18.900 & 64.179 & $2.3 \pm 0.2$ & $118.54 \pm 1.42$ & 40.61 \\
\hline $150330,02: 12: 43$ & -18.923 & 64.218 & $2.8 \pm 0.2$ & $119.31 \pm 2.31$ & 42.88 \\
\hline $150331,01: 04: 36$ & -18.929 & 64.211 & $2.3 \pm 0.3$ & $118.32 \pm 2.27$ & 42.88 \\
\hline $150331,16: 30: 29$ & -18.903 & 64.181 & $2.3 \pm 0.1$ & $118.37 \pm 2.52$ & 40.77 \\
\hline $150406,20: 25: 42$ & -18.870 & 64.156 & $3.0 \pm 0.3$ & $119.55 \pm 2.11$ & 38.67 \\
\hline $150412,16: 15: 36$ & -18.917 & 64.190 & $2.4 \pm 0.2$ & $117.84 \pm 1.78$ & 41.64 \\
\hline 150416,00:51:55 & -18.917 & 64.190 & $2.2 \pm 0.2$ & $117.83 \pm 1.67$ & 41.64 \\
\hline $150422,02: 36: 21$ & -18.891 & 64.156 & $2.1 \pm 0.3$ & $117.73 \pm 2.73$ & 39.41 \\
\hline $150425,22: 12: 51$ & -18.886 & 64.161 & $1.6 \pm 0.1$ & $118.47 \pm 2.18$ & 39.41 \\
\hline $150604,12: 38: 18$ & -18.874 & 64.166 & $2.3 \pm 0.2$ & $120.64 \pm 1.73$ & 39.62 \\
\hline $150730,02: 43: 14$ & -18.925 & 64.211 & $2.2 \pm 0.3$ & $118.58 \pm 2.04$ & 42.71 \\
\hline $151001,19: 22: 45$ & -18.882 & 64.164 & $2.2 \pm 0.0$ & $119.09 \pm 1.46$ & 39.41 \\
\hline \multicolumn{6}{|l|}{ Cluster 2} \\
\hline 150212,22:08:04 & -19.076 & 62.273 & $2.2 \pm 0.1$ & $141.80 \pm 2.99$ & 299.66 \\
\hline $150217,21: 43: 41$ & -19.117 & 62.277 & $2.4 \pm 0.3$ & $139.22 \pm 1.86$ & 298.12 \\
\hline $150217,22: 36: 07$ & -19.112 & 62.290 & $1.8 \pm 0.1$ & $138.24 \pm 2.54$ & 298.57 \\
\hline \multicolumn{6}{|l|}{ Cluster 3} \\
\hline 141125,00:53:54 & -22.083 & 64.061 & $3.3 \pm 0.2$ & $271.46 \pm 2.61$ & 166.50 \\
\hline 141127,01:57:58 & -22.093 & 64.063 & $3.5 \pm 0.2$ & $272.58 \pm 2.88$ & 166.50 \\
\hline 150419,17:46:08 & -22.086 & 64.006 & $3.0 \pm 0.2$ & $270.49 \pm 2.78$ & 167.69 \\
\hline 150508,07:19:32 & -22.006 & 64.042 & $3.3 \pm 0.3$ & $262.87 \pm 3.42$ & 166.21 \\
\hline 151012,20:47:05 & -21.948 & 64.092 & $2.8 \pm 0.2$ & $257.66 \pm 2.97$ & 165.02 \\
\hline $151130,22: 27: 54$ & -21.952 & 64.108 & $2.9 \pm 0.2$ & $258.56 \pm 4.60$ & 164.69 \\
\hline
\end{tabular}

Table S2. Details of the events in Clusters 1, 2 and 3. 